\title{
Traditional Chinese medicine Yisui Tongjing relieved neural severity in experimental autoimmune neuritis rat model
}

This article was published in the following Dove Press journal:

Neuropsychiatric Disease and Treatment

29 September 2016

Number of times this article has been viewed

\section{Erli Zhang' \\ Mingquan $\mathrm{Li}^{2}$ \\ Jianjun Zhao ${ }^{2}$ \\ Yuxiang Dong' \\ Xueqin Yang' \\ Jingbo Huang'}

'Department of Traditional Chinese Medicine, The First Bethune Hospital of Jilin University, ${ }^{2}$ Department of Encephalopathy, The First Affiliated Hospital of Changchun University of Traditional Chinese Medicine, Changchun, People's Republic of China
Correspondence: Erli Zhang Department of Traditional Chinese Medicine, The First Bethune Hospital of Jilin University, No 7I Xinmin Street, Changchun I3002I, People's Republic of China Tel +86 I58 04300575 Email zhangerlilII7@I63.com
Objective: To study the effect of Yisui Tongjing (YSTJ) prescription on motor nerve conduction velocity (MNCV) and microstructure of the sciatic nerve in experimental autoimmune neuritis (EAN) rats, the Guillain-Barré syndrome classic animal models.

Materials and methods: In this study, we established an EAN model in Lewis rats by immunization. We evaluated the potential clinical application of a traditional Chinese medicine YSTJ by intragastric administration and compared its effect with immunoglobulin. The sciatic MNCV was measured by electrophysiology experiment. Hematoxylin-eosin staining and transmission electron microscope analysis were used to determine the pathologically morphological changes before and after YSTJ application.

Results: We found that application of YSTJ could significantly alleviate the clinical signs in EAN rats. The treatment also increased MNCV in the sciatic nerve compared to that in the untreated nerve. Demyelination in the sciatic nerve in EAN rats was significantly ameliorated, and newly generated myelinated nerve fibers were observed with treatment of high dose of YSTJ. Conclusion: This study showed that the traditional Chinese medicine YSTJ was likely to serve as a therapeutic medicine in autoimmune neuropathies, providing an effective and economic means to the treatment of Guillain-Barré syndrome.

Keywords: Yisui Tongjing, experimental autoimmune neuritis, motor nerve conduction velocity, sciatic nerve, demyelination

\section{Introduction}

Guillain-Barré syndrome (GBS) is an inflammatory demyelinating disease of the human peripheral nervous system (PNS), which is characterized by a rapid-onset muscle weakness caused by the immune system. Symptoms of GBS include sensory disturbance and loss of deep tendon reflexes in the extremities. ${ }^{1}$ GBS affects all age groups, with a yearly incidence rate between 1.1 and 1.8 per $100,000 .^{2,3}$ The pathogenesis of GBS is well characterized and involves an autoimmune postinfectious response in most cases. ${ }^{4-6}$ The most commonly reported pathologies of GBS were demyelination and axonal degeneration. ${ }^{7}$ At present, the main treatments of GBS include plasma exchange and intravenous immunoglobulin (IVIg). ${ }^{8}$ In spite of these optimal treatments, there are still significant mortality and morbidity due to the inefficiency to some patients.

Experimental autoimmune neuritis (EAN) is an acute demyelinating inflammatory disease of the PNS. EAN can be induced in animal models by immunization with various PNS autoantigens in conjunction with complete Freund's adjuvant. ${ }^{9}$ Pathologically, EAN is characterized by inflammatory cell infiltration, disruption of 
blood-nerve barrier, and demyelination of PNS. Since EAN has an innate ability to mimic many clinical, histological, and immunological characteristics of GBS, it is considered as an excellent model for investigating the pathogenesis of GBS and assessing new therapies. ${ }^{10}$

Recently, some traditional medicines were found to be effective in GBS treatment, including bee venom ${ }^{11}$ and intragastric application of berberine. ${ }^{12}$ These studies demonstrated the unique clinical effect of some traditional medicines. However, the advantage of these traditional medicines has not been evaluated yet, and the clinical mechanism of these medicines is still unknown to us. In this study, we evaluated the clinical effect of a traditional Chinese medicine known as Yisui Tongjing (YSTJ; literally meaning nourishing marrow and dredging channels) in the EAN rat model. YSTJ is a widely used term in traditional Chinese Medicine. Generally, it is a therapeutic method and is a traditional medicine prescription. According to the traditional Chinese medicine theory, sui means the marrow and jing means the main and collateral channels, both of which play a role in the human motor function. Clinically, YSTJ is used to treat limb paralysis and muscle weakness and improve the motor function. Based on the data obtained in this study, we concluded that YSTJ may serve as an effective medicine candidate for the treatment of GBS.

\section{Materials and methods}

\section{Animals}

Adult female Lewis rats aged 10-12 weeks and weighing 180-200 g were purchased from Vital River Laboratories Co., Ltd. (Beijing, People's Republic of China). All procedures of animal handling were carried out in accordance with the protocols of the animal care guidelines of the Institutional Animal Care and Use Committee of Jilin University which approved the study.

\section{EAN rat model and assessment of neurological deficit}

Establishment of the EAN rat model has been described previously. ${ }^{13}$ In brief, EAN rats were induced by subcutaneous injection at hind feet with $100 \mu \mathrm{L}$ of an emulsion containing $200 \mu \mathrm{g} \mathrm{P} 0_{180-199}$ peptide (GL Biochem Shanghai, People's Republic of China), $1 \mathrm{mg}$ Bacillus Calmette-Guérin vaccine (Shanghai Institute of Biological Products Shanghai, People's Republic of China), and $1 \mathrm{mg}$ Mycobacterium tuberculosis (Harbin Pharmaceutical Group Harbin, People's Republic of China). Rats were scored at 10 days, 18 days, and 25 days after immunization for development of EAN as follows: $0=$ normal, $1=\operatorname{limp}$ tail, $2=$ mild paresis of the hind limbs, $3=$ severe paraparesis or paraplegia of the hind limbs, and $4=$ tetraparesis.

\section{Grouping}

Animals were randomly divided into six groups, including normal (blank control) group, the model group, intravenous IVIg treatment group, high dose $(2.0 \mathrm{~mL} / \mathrm{d})$, medium dose $(1.5 \mathrm{~mL} / \mathrm{d})$, and low dose $(1 \mathrm{~mL} / \mathrm{d})$ of YSTJ prescription groups. Each group contained five to ten rats. IVIg treatment started at day 11 after immunization for five consecutive doses (480 mg, one dose per day). YSTJ prescription started at day 11 after immunization by intragastric administration.

\section{Measurement of sciatic motor nerve conduction velocity}

Five rats randomly selected from each group were used to measure the sciatic nerve conduction velocity (NCV) at 1,2, and 4 weeks after treatment. Rats were anesthetized by $10 \%$ chloral hydrate injection intraperitoneally $(0.3 \mathrm{~mL} / 100 \mathrm{mg})$. Surgery was performed on the left hind limb of anesthetized rats. The skin was incised and detached between the biceps femoris and semitendinosus muscles to expose the sciatic nerve, to which three electrodes were attached, including one stimulating electrode and two recording electrodes. The sciatic nerve was stimulated by the stimulating electrode, and conduction was detected as action potentials by two recording electrodes. Motor nerve conduction velocity (MNCV) was measured according to the following equation: MNCV $(\mathrm{m} / \mathrm{s})=$ distance between two recording electrode/time difference between two action potentials. The stimulus was digitized and captured with the BL-420 biological functional system (Chengdu Taimeng Technology Co.,Ltd., Chengdu, People's Republic of China). The sciatic nerve was stimulated with constant voltage $(3 \mathrm{~V})$ square-wave pulses $(0.1 \mathrm{~ms})$.

\section{Hematoxylin-eosin staining}

Sciatic nerve was fixed in formaldehyde calcium for 48 hours, dehydrated in ethanol, and embedded in paraffin. Longitudinal sections of sciatic nerves $(5 \mu \mathrm{m})$ were stained with hematoxylin-eosin for evaluating the morphology of nerve fibers. Briefly, sections were deparaffinized in xylene and then stained with hematoxylin for 45 seconds. After rinsing with distilled water, sections were stained with eosin for 15 seconds. The images were captured using an ECLIPSE 90i (Nikon Corporation, Tokyo, Japan).

\section{Transmission electron microscope}

Sciatic nerves were prefixed with $2.5 \%$ glutaraldehyde for 1.5 hours and postfixed with $1 \%$ osmic acid for 1.5 hours. Then, tissues were dehydrated with ethanol-acetone. Samples were embedded in Epon812 ethoxyline resin. Sections were stained for 20 minutes with $2 \%(\mathrm{w} / \mathrm{v})$ aqueous uranyl 
acetate followed by 6 minutes with Reynolds lead citrate, which were later on examined in a JEM-1200EX transmission electron microscope.

\section{Statistical analysis}

Raw data were analyzed using SPSS16.0 software (SPSS Inc., Chicago, IL, USA). All data were presented as mean $\pm \mathrm{SD}$. The difference among all the groups was determined by unpaired $t$-test and one-way analysis of variance. A $P$-value of $<0.05$ was considered as significantly different and $<0.01$ was considered as very significantly different, while a $P$-value $>0.05$ was considered as not significantly different.

\section{Result}

\section{YSTJ treatment alleviated the clinical signs in EAN rats}

The first neurological sign (limb tail) of EAN rats was observed on the seventh to ninth day after immunization. The neurological severity of EAN increased rapidly over time and reached a maximum on the 17 th day with a maximal score. On the tenth day after immunization (pretreatment stage), the clinical score in the model group was significantly higher than that in the control group (Figure 1, $P<0.05$ ). On the 18 th day after immunization (1 week after treatment), the clinical score in all the treatment groups did not show significant change compared with that of the model group (Figure 1). In contrast, 2 weeks after treatment, the clinical score in the IVIg group and the high dose of YSTJ (H-YSTJ) prescription group significantly decreased compared with that of the model group (Figure $1, P<0.05$ ). However, there was no significant clinical score change in the low- and mediumdose YSTJ (L-YSTJ and M-YSTJ) groups.

\section{YSTJ treatment recovered sciatic MNCV in $\mathrm{EAN}$ rats}

After the immunization, sciatic NCV significantly decreased in the IVIg and H-YSTJ groups compared with the control group (pretreatment in Figure 2, $P<0.05$ ). One week after the treatment, the MNCV increased in the IVIg and H-YSTJ groups (Figure 2, $P<0.05$ ). However, no significant change in MNCV was found in the L-YSTJ and M-YSTJ groups compared with the model group. Besides, all four treatment groups showed significant increases in MNCV compared with the model group at 2 weeks after the treatment (Figure 2, $P<0.05)$. The effect of H-YSTJ and M-YSTJ was close to that of IVIg. Four weeks after treatment, all four treatment groups showed a significantly positive effect on the MNCV recovery. Furthermore, a dose-dependent relationship formed within three YSTJ groups (Figure 2). All the statistic data are presented in Table 1.

\section{YSTJ treatment prevented demyelination in sciatic nerve}

Next, we used hematoxylin-eosin staining to determine the morphological changes in sciatic nerve after YSTJ

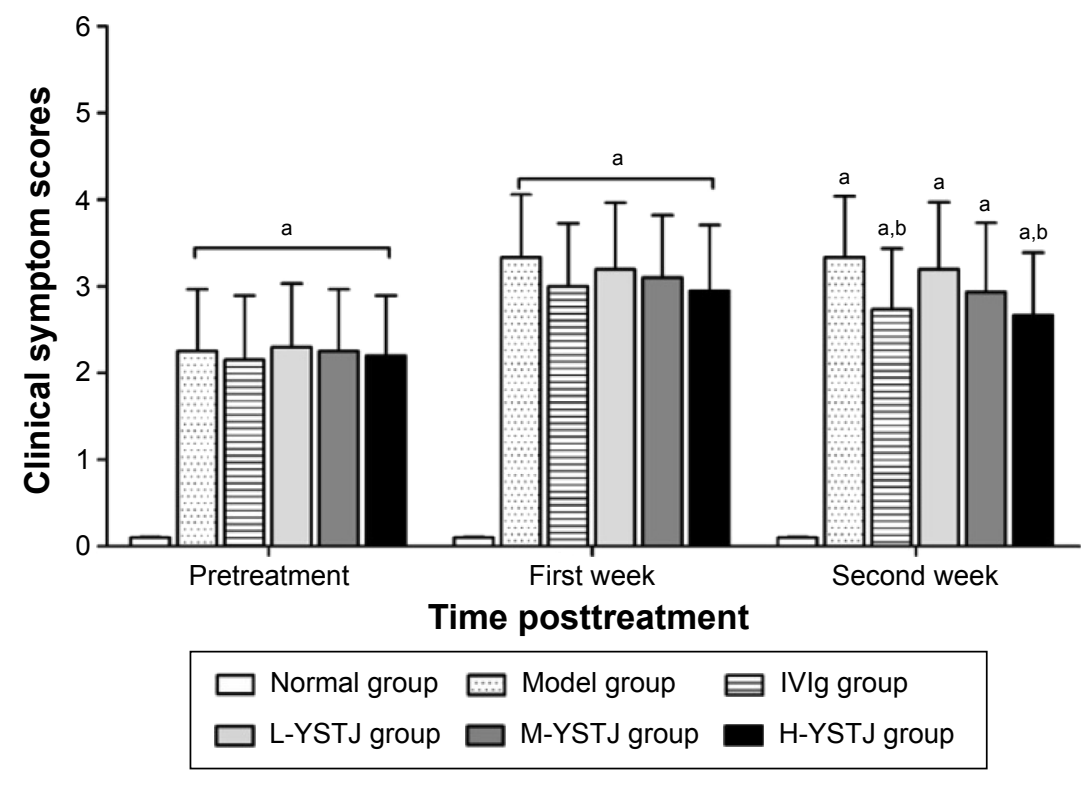

Figure I Comparison of the clinical score among different groups.

Notes: "a" represents the significant difference compared with the normal (blank control) group $(P<0.05)$. "b" represents the significant difference compared with the model group $(P<0.05)$. $n=10$ in each group.

Abbreviations: IVlg, immunoglobulin; YSTJ, Yisui Tongjing; H-YSTJ, high dose of YSTJ; M-YSTJ, medium dose of YST]; L-YSTJ, low dose of YSTJ. 


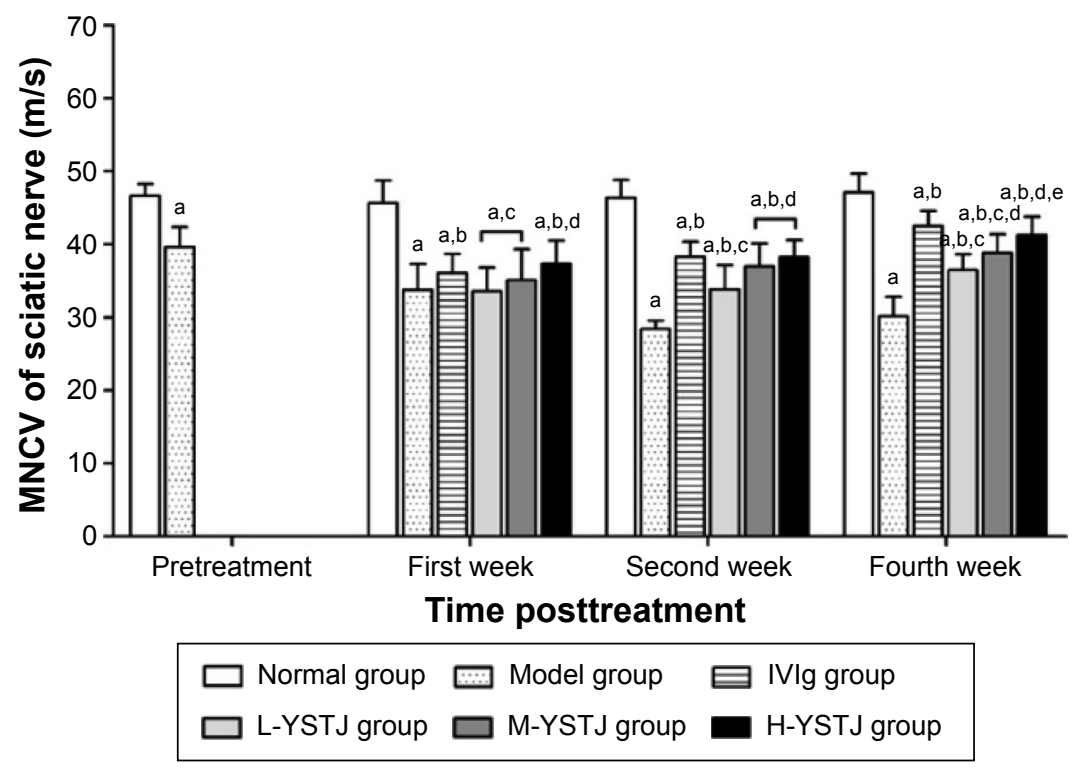

Figure 2 Comparison of the MNCV of sciatic nerve among different groups.

Notes: "a" represents the significant difference compared with the normal (blank control) group $(P<0.05)$. "b" represents the significant difference compared with the model group $(P<0.05)$. "c" represents the significant difference compared with the IVIg group $(P<0.05)$. "d" represents the significant difference compared with the L-YSTJ group $(P<0.05)$. "e" represents the significant difference compared with the M-YSTJ group $(P<0.05)$. $n=5$ in each group.

Abbreviations: MNCV, motor nerve conduction velocity; IVlg, immunoglobulin; YSTJ, Yisui Tongjing; H-YSTJ, high dose of YSTJ; M-YSTJ, medium dose of YST]; L-YSTJ, low dose of YSTJ.

treatment. In the normal group, axons were aligned orderly and surrounded by myelin sheath. The blue-stained nuclei of Schwann cells were clear (Figure 3A). In contrast, obvious demyelination was observed in the model group (Figure 3C). With the treatment of H-YSTJ, the nerve fiber structure was generally normal. There was no apparent demyelination in the sciatic nerve (Figure 3G). Similar structural recovery was observed in the IVIg group (Figure 3E).

We also observed the ultrastructural changes using a transmission electron microscope. In the control group, the axon structure of nerve fiber was normal, with neurofilament and mitochondria in the cytoplasm. Myelin sheath was aligned as concentric circles (Figure 3B). However,

Table I MNCV of sciatic nerves in each group after treatment

\begin{tabular}{|c|c|c|c|}
\hline Group & $\begin{array}{l}0 \text { day after } \\
\text { treatment }\end{array}$ & $\begin{array}{l}7 \text { days after } \\
\text { treatment }\end{array}$ & $\begin{array}{l}2 I \text { days after } \\
\text { treatment }\end{array}$ \\
\hline & n MNCV & n MNCV & n MNCV \\
\hline Normal & $545.66 \pm 3.08$ & $5 \quad 46.38 \pm 2.46$ & $5 \quad 47.08 \pm 2.59$ \\
\hline Model & $533.74 \pm 3.58^{* *}$ & $\begin{array}{ll}5 & 28.39 \pm 1.17^{* *}\end{array}$ & $430.14 \pm 2.69 * *$ \\
\hline IVlg & $536.05 \pm 2.65^{* *, \Delta \Delta}$ & $538.27 \pm 2.06 * *, \Delta \Delta$ & $542.52 \pm 2.08^{* *, \Delta \Delta}$ \\
\hline H-YSTJ & $537.35 \pm 3.15^{* *, \Delta \Delta, \mathbf{\Lambda}}$ & $538.23 \pm 2.34^{* *, \Delta \Delta, \boldsymbol{V}}$ & $541.27 \pm 2.52^{* *, \Delta \Delta, \nabla}$ \\
\hline M-YSTJ & $535.11 \pm 4.19 * *, \Delta \Delta, \Delta \Lambda$ & $536.95 \pm 3.18^{* *, \Delta \Delta, \Delta \Delta}$ & $538.84 \pm 2.55^{* *, \Delta \Delta, \Delta}$ \\
\hline L-YSTJ & $533.56 \pm 3.28 * *, \Delta, \Delta$ & $533.81 \pm 3.32^{* *, \Delta \Lambda, \Delta \Delta}$ & $536.46 \pm 2.20 * *, \Delta \Delta, \Delta \Delta$ \\
\hline
\end{tabular}

Notes: $* * P<0.0$ I (compared with the normal group); $\triangle \Delta P<0.01$ (compared with the model group); and $\Delta P<0.05, \Delta \Delta P<0.01, \nabla P>0.05$ (compared with the IVlg group). Abbreviations: MNCV, motor nerve conduction velocity; IVIg, immunoglobulin; YST], Yisui Tongjing; H-YSTJ, high dose of YSTJ; M-YST], medium dose of YST]; L-YST], low dose of YSTJ. folds and crinkles of myelin sheath and wide gaps between axon and sheath appeared in the model group (Figure 3D). Furthermore, demyelination and myelinolysis were obvious, and axons and mitochondria were lost (Figure 3D). In the presence of H-YSTJ, fewer folds and crinkles of myelin sheath were observed. Newly generated myelinated nerve fibers were aligned orderly (Figure $3 \mathrm{H}$ ). Similar results were found in the IVIg group (Figure 3F). Collectively, demyelination and other morphological defects in the sciatic nerve induced by EAN were significantly ameliorated with the treatment of H-YSTJ.

\section{Discussion}

In the present study, we found that a traditional Chinese medicine YSTJ had beneficiary effect on the sciatic nerve in the EAN rat model. The application of this medicine was able to relieve the clinical symptoms. Besides, it could restore (at least partially) the physiological function of sciatic nerve. Furthermore, the pathological impairment in axonal morphology was restored in the presence of YSTJ prescription. Collectively, these results indicated that YSTJ was a promising medicine to treat the human GBS.

The symptoms of GBS include muscle weakness and tetraparesis, which are close to the flaccidity disease in traditional Chinese medical science. YSTJ consists of tortoise plastron, strychnos, and pilose antler. YSTJ has been used to treat the flaccidity disease and has a good clinical effect. 

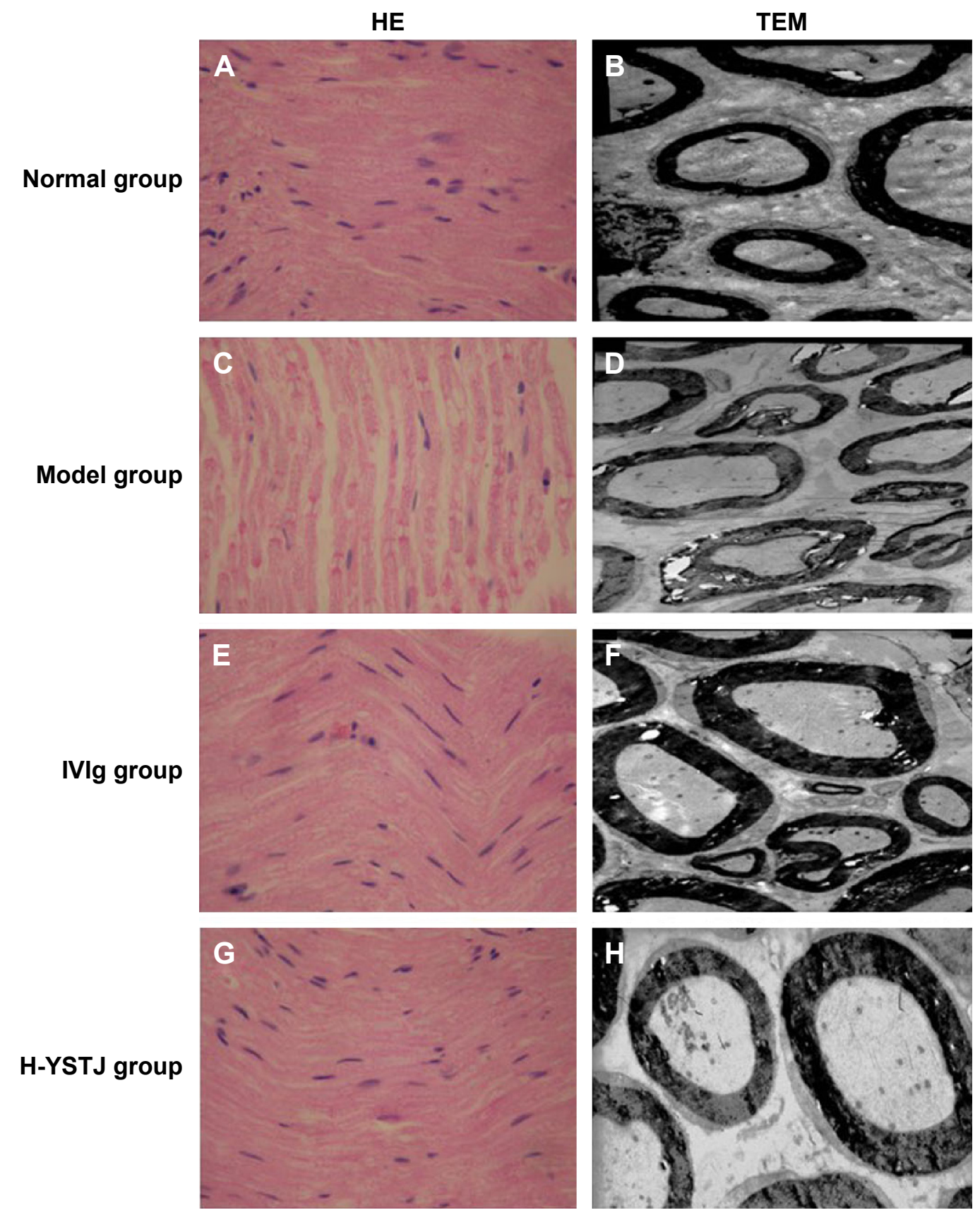

Figure 3 The morphological change in sciatic nerve shown by HE staining (A, C, E, G, 400x) and observed by TEM (B, D, F, H, 500x).

Abbreviations: HE, Hematoxylin-eosin; TEM, transmission electron microscope; IVlg, immunoglobulin; H-YST], high dose of Yisui Tongjing.

Tortoise plastron contains fatty acids, fatty acid esters, and steroids. It was recently reported that tortoise plastron could induce the neuronal differentiation from transplanted mesenchymal stem cell in cerebral ischemia model rats. ${ }^{14}$ This study provided evidence to support the conclusion that tortoise plastron promoted the repair of neural damage. The active ingredient of strychnos is alkaloid, which plays a vital role in the excitation of spinal reflex. ${ }^{15}$ Therefore, strychnos has been applied to treat flaccid paralysis. Pilose antler contains lipids and polypeptides. Antler polypeptide could promote sciatic nerve regeneration in rats ${ }^{16}$ and motor function recovery in spinal cord injury rats. ${ }^{17}$ Collectively, all these results provided experimental basis that YSTJ prescription has the functions of spinal cord excitation, providing the essential growth factors to nerve, and promoting neural recovery and regeneration. Therefore, YSTJ prescription is beneficiary to relieve the symptom of GBS and promote the recovery of the disease. 
According to the difference in neuroelectrophysiological and neuropathological manifestations, there are two types of GBS including demyelinating and axonal variants. ${ }^{18}$ The characteristic of demyelinated GBS is the decreasing NCV and distal latency delay, but the normal distal compound muscle action potential. In contrast, the axonal subtype is featured with impaired or even loss of distal compound muscle action potential. In EAN animal models, immunization with myelin protein leads to pathological symptom similar to demyelinating GBS, while immunization with jejuni or ganglioside causes symptom similar to axonal subtype. ${ }^{19}$ In the present study, we immunized the animal with $\mathrm{P}_{0}$ polypeptide to establish the EAN model, and demyelination was observed in the sciatic nerve. Thus, we used NCV to evaluate the neural severity in the animal model. NCV is an important aspect of nerve conduction studies. Conduction velocity depends largely on the degree to which an axon is myelinated. ${ }^{20}$ In the sciatic nerve, a fall in MNCV is characteristic of demyelinating neuropathies. ${ }^{21}$ This was observed in the EAN rat model that we have established (Figure 2). Generally, the treatment of GBS is the intravenous injection of IVIg. ${ }^{22,23}$ However, a standard dose of IVIg was not sufficiently effective in many GBS patients so that a second or multiple doses were needed. ${ }^{23}$ This decreased the medicine efficiency and increased the economic burden for patients. In our study, we compared the clinical effect of YSTJ with IVIg. All three different doses showed significant recovery in MNCV in the animal model. Besides, the effect of high dose was comparable with the IVIg treatment (Figure 2). This indicated the potent clinical efficiency of YSTJ and increased the possibility to replace the IVIg with a more economical prescription.

A previous study found that there were two kinds of myelin sheath change in the EAN animal model, including a vesicular disorganization of the myelin lamellae and a separation of the myelin lamellae. ${ }^{24}$ Then, a close correlation between axonal damage and autoimmune neuritis was clarified. ${ }^{25,26}$ Previous reports indicated that the pathological changes in autoimmune neuritis include inflammatory cell filtration and demyelination. ${ }^{27,28}$ Besides the abovementioned description, our model showed folds and crinkles of myelin sheath, wide gaps between axon and sheath, and total myelinolysis and mitochondrial loss in the EAN rats (Figure 3). More importantly, all these morphological defects were recovered by YSTJ prescription. This further proved the potential clinical application in the treatment of autoimmune neuritis at the histological level.

Collectively, this study showed that traditional Chinese medicine YSTJ effectively prevented progression and facilitated recovery from autoimmune neuritis disease in the EAN rat model clinically, electrophysiologically, and histologically. This provided strong evidence to support the conclusion that YSTJ could potentially be a potent medicine in the treatment of GBS, contributing to the development of more effective and less costly treatment for autoimmune peripheral neuropathies. Further study should be focused on the molecular mechanism of the clinical effect of this traditional Chinese medicine in the EAN animal models and the research on the clinical test for YSTJ prescription on the human patients of GBS.

\section{Acknowledgment}

This study was supported by the Specialized Research Fund for the Doctoral Program of Higher Education of China (Grant no 20092227110003).

\section{Disclosure}

The authors report no conflicts of interest in this work.

\section{References}

1. Hughes RAC, Cornblath DR. Guillain-Barre syndrome. Lancet. 2005; 366(9497):1653-1666.

2. McGrogan A, Madle GC, Seaman HE, de Vries CS. The epidemiology of Guillain-Barre syndrome worldwide. A systematic literature review. Neuroepidemiology. 2009;32(2):150-163.

3. Rajabally YA. Treatment of Guillain-Barre syndrome: a review. Inflamm Allergy Drug Targets. 2012;11(4):330-334.

4. Israeli E, Agmon-Levin N, Blank M, Chapman J, Shoenfeld Y. GuillainBarre syndrome - a classical autoimmune disease triggered by infection or vaccination. Clin Rev Allergy Immunol. 2012;42(2):121-130.

5. Nussinovitch U, Shoenfeld Y. The role of gender and organ specific autoimmunity. Autoimmun Rev. 2012;11(6-7):A377-A385.

6. Shoenfeld Y, George J, Peter JB. Guillain-Barre as an autoimmune disease. Int Arch Allergy Imm. 1996;109(4):318-326.

7. Peric S, Milosevic V, Berisavac I, et al. Clinical and epidemiological features of Guillain-Barre syndrome in the Western Balkans. J Peripher Nerv Syst. 2014;19(4):317-321.

8. Aronovich R, Katzav A, Chapman J. The strategies used for treatment of experimental autoimmune neuritis (EAN): a beneficial effect of glatiramer acetate administered intraperitoneally. Clin Rev Allergy Immunol. 2012;42(2):181-188.

9. Pineda AAM, Minohara M, Kawamura N, et al. Preventive and therapeutic effects of the selective Rho-kinase inhibitor fasudil on experimental autoimmune neuritis. $J$ Neurol Sci. 2011;306(1-2):115-120.

10. Kafri M, Drory VE, Wang NS, Rabinowitz R, Korczyn AD, Chapman J. Assessment of experimental autoimmune neuritis in the rat by electrophysiology of the tail nerve. Muscle Nerve. 2002;25(1):51-57.

11. Lee HJ, Park IS, Lee JI, Kim JS. Guillain-Barre syndrome following bee venom acupuncture. Intern Med. 2015;54(8):975-978.

12. Li H, Li XL, Zhang M, et al. Berberine ameliorates experimental autoimmune neuritis by suppressing both cellular and humoral immunity. Scand J Immunol. 2014;79(1):12-19.

13. Zhang ZY, Zhang Z, Fauser U, Schluesener HJ. Expression of interleukin-16 in sciatic nerves, spinal roots and spinal cords of experimental autoimmune neuritis rats. Brain Pathol. 2009;19(2):205-213.

14. Du SH, Chen DF, Li YW, et al. [Effect of tortoise plastron on cell differential of neurons after mesenchymal stem cell transplantation in cerebral ischemia in rats]. Zhonghua Yi Xue Za Zhi. 2005;85(3):205-207. Chinese. 
15. Wu M, Fang M, Hu Y, Wang X. Four types of traditional Chinese medicine inducing epileptic seizures. Seizure. 2012;21(5):311-315.

16. Lu L, Wang K, Li L, Xuan Z, Gong X. [Effect of velvet antler polypeptide on peripheral nerve regeneration]. Zhongguo Xiu Fu Chong Jian Wai Ke Za Zhi. 2008;22(12):1458-1461. Chinese.

17. Li ZH, Leng XY, Gao ZL. [Protective effect of velvet antler polypeptide (VAP) on rats with the spinal cord injury]. Zhongguo Gu Shang. 2008;21(4):285-286. Chinese.

18. Yuki N, Hartung HP. Guillain-Barre syndrome. N Engl J Med. 2012; 366(24):2294-2304

19. Shin T, Ahn M, Matsumoto Y, Moon C. Mechanism of experimental autoimmune neuritis in Lewis rats: the dual role of macrophages. Histol Histopathol. 2013;28(6):679-684.

20. Niknami M, Wang MX, Nguyen T, Pollard JD. Beneficial effect of a multimerized immunoglobulin $\mathrm{Fc}$ in an animal model of inflammatory neuropathy (experimental autoimmune neuritis). J Peripher Nerv Syst. 2013;18(2):141-152.

21. Lin HH, Spies JM, Lu JL, Pollard JD. Effective treatment of experimental autoimmune neuritis with human immunoglobulin. J Neurol Sci. 2007;256(1-2):61-67.
22. Hughes RA, Swan AV, van Doorn PA. Intravenous immunoglobulin for Guillain-Barre syndrome. Cochrane Database Syst Rev. 2012;7: CD002063.

23. van Doorn PA, Kuitwaard K, Walgaard C, van Koningsveld R, Ruts L, Jacobs BC. IVIG treatment and prognosis in Guillain-Barre syndrome. J Clin Immunol. 2010;30(suppl 1):S74-S78.

24. Allt $\mathrm{G}$. The node of Ranvier in experimental allergic neuritis: an electron microscope study. J Neurocytol. 1975;4(1):63-76.

25. Mathey EK, Park SB, Hughes RA, et al. Chronic inflammatory demyelinating polyradiculoneuropathy: from pathology to phenotype. J Neurol Neurosurg Psychiatry. 2015;86(9):973-985.

26. Hadden RD, Hughes RA. Management of inflammatory neuropathies. J Neurol Neurosurg Psychiatry. 2003;74(suppl 2):ii9-ii14.

27. Asbury AK, Arnason BG, Adams RD. The inflammatory lesion in idiopathic polyneuritis. Its role in pathogenesis. Medicine (Baltimore). 1969;48(3):173-215.

28. Taylor JM, Pollard JD. Neurophysiological changes in demyelinating and axonal forms of acute experimental autoimmune neuritis in the Lewis rat. Muscle Nerve. 2003;28(3):344-352.
Neuropsychiatric Disease and Treatment

\section{Publish your work in this journal}

Neuropsychiatric Disease and Treatment is an international, peerreviewed journal of clinical therapeutics and pharmacology focusing on concise rapid reporting of clinical or pre-clinical studies on a range of neuropsychiatric and neurological disorders. This journal is indexed on PubMed Central, the 'PsycINFO' database and CAS,

\section{Dovepress}

and is the official journal of The International Neuropsychiatric Association (INA). The manuscript management system is completely online and includes a very quick and fair peer-review system, which is all easy to use. Visit http://www.dovepress.com/testimonials.php to read real quotes from published authors. 